\title{
Outcomes of Patients With Advanced Gastrointestinal Cancer in Relationship to Opioid Use: Findings From Eight Clinical Trials
}

\author{
Omar Abdel-Rahman, MD ${ }^{1}$; Hatim Karachiwala, MD ; and Jacob C. Easaw, MD ${ }^{1}$
}

\begin{abstract}
Background: This study assessed the patterns of opioid use among patients with advanced gastrointestinal cancers who were included in 8 clinical trials and evaluated the impact of opioid use on survival outcomes of included patients. Methods: Deidentified datasets from 8 clinical trials evaluating first-line systemic treatment of advanced gastrointestinal cancers were accessed from the Project Data Sphere platform (ClinicalTrial.gov identifiers: NCT01124786, NCT00844649, NCT00290966, NCT00678535, NCT00699374, NCT00272051, NCT00305188, and NCT00384176). These trials evaluated patients with pancreatic carcinoma, gastric carcinoma, hepatocellular carcinoma (HCC), and colorectal carcinoma. Multivariable logistic regression analysis was used to evaluate factors predicting the use of opioids. Kaplan-Meier survival estimates were used to compare survival outcomes in each disease entity among patients who did or did not receive opioid treatment. Multivariable Cox regression analysis was then used to further assess the impact of opioid use on survival outcomes in each disease entity. Results: A total of 3,441 participants were included in the current analysis. The following factors predicted a higher probability of opioid use within logistic regression analysis: younger age at diagnosis (odds ratio [OR], 0.990; $95 \% \mathrm{Cl}, 0.984-0.997 ; P=.004$ ), nonwhite race (OR for white vs nonwhite, $0.749 ; 95 \% \mathrm{Cl}, 0.600-0.933 ; P=.010)$, higher ECOG score (OR for 1 vs $0,1.751 ; 95 \% \mathrm{Cl}, 1.490-2.058 ; P<.001$ ), and pancreatic primary site (OR for colorectal vs pancreatic, 0.241 ; $95 \% \mathrm{Cl}, 0.198-0.295$; $P<.001)$. Use of opioids was consistently associated with worse overall survival (OS) in Kaplan-Meier survival estimates of each disease entity ( $P=.008$ for pancreatic cancer; $P<.001$ for gastric cancer, HCC, and colorectal cancer). In multivariable Cox regression analysis, opioid use was associated with worse OS among patients with pancreatic cancer (hazard ratio [HR], 1.245; 95\% Cl, 1.063-1.459; $P=.007$ ), gastric cancer $(\mathrm{HR}, 1.725 ; 95 \% \mathrm{Cl}, 1.403-2.122 ; P<.001), \mathrm{HCC}(\mathrm{HR}, 1.841 ; 95 \% \mathrm{Cl}$, $1.480-2.290 ; P<.001)$, and colorectal cancer $(\mathrm{HR}, 1.651 ; 95 \% \mathrm{Cl}$, $1.380-1.975 ; P<.001)$. Conclusions: Study findings suggest that opioid use is consistently associated with worse OS among patients with different gastrointestinal cancers. Further studies are needed to understand the underlying mechanisms of this observation and its potential implications.
\end{abstract}

J Natl Compr Canc Netw 2020;18(5):575-581 doi: $10.6004 /$ jnccn.2019.7382

${ }^{1}$ Department of Oncology, University of Alberta, Edmonton, Alberta, Canada.

\section{Background}

Opioid analgesia is an integral part of pain management among patients with advanced cancer. ${ }^{1,2}$ A potential correlation between opioid analgesics and cancer progression has been previously hypothesized. ${ }^{3,4}$ However, most of the published data evaluating this correlation have focused on the impact of perioperative opioid anesthetics on cancer recurrence and progression., These studies were confounded, however, by small sample size, retrospective data collection (with the potential of missing data or bias), and little attention to patients with advanced disease who received opioids for pain management rather than as a perioperative anesthetic. To provide a better assessment of the impact of opioid use on outcomes of patients with advanced cancer, this question needs to be addressed through a prospectively collected dataset using a meticulous gathering of patient and treatment data to minimize the risk of bias and confounding factors.

Project Data Sphere is a recently launched platform that allows researchers access to deidentified clinical trial datasets. ${ }^{7}$ It thus represents an excellent opportunity to examine this question with limited confounders. To minimize heterogeneity of the sample used for this analysis, we focused only on patients with advanced gastrointestinal cancer. Our working hypothesis was that patients with advanced gastrointestinal cancers who received opioids have poor overall survival (OS) and progression-free survival (PFS). The aims of this study were to assess the patterns of opioid use among patients with advanced gastrointestinal cancers who were included in 8 clinical trials and to evaluate the impact of opioid use on survival outcomes of those patients.

\section{Methods}

\section{Study Cohort}

The current study represents a pooled analysis of 8 clinical trials including patients with advanced gastrointestinal 
cancers treated using first-line systemic therapy. Specifically, 2 of these trials included patients with advanced pancreatic cancer (ClinicalTrials.gov identifiers: NCT01124786, NCT00844649), 2 included patients with advanced gastric cancer (NCT00290966, NCT00678535), 1 included patients with advanced hepatocellular carcinoma (HCC; NCT00699374), and 3 included patients with advanced colorectal cancer (NCT00272051, NCT00305188, and NCT00384176). Additional details about each of these trials, including methodology and treatment arms, are provided in Table 1. For 6 of these trials, primary results were published previously. ${ }^{8-13}$ For the NCT01124786 study, both study arms were included, whereas for the remaining 7 studies, only active comparator arms were included.

\section{Data Collection}

For each included participant, the following data were collected (when available): age at diagnosis, body mass index (BMI), race, sex, ECOG performance status (PS), primary tumor site, number of metastatic sites, and treatment using any opioid medication. Opioid use data evaluated in the current analysis were restricted in most of the studies to opioid use concurrent with first-line systemic therapy administration. For patients in the HCC study, information about extrahepatic spread and macrovascular invasion was also included.

\section{Endpoints}

Co-primary endpoints of the current study were OS (defined as time from randomization until death of any cause) and PFS (defined as time from randomization until death or disease progression).

\section{Statistical Analyses}

Chi-square testing was initially used to compare baseline categorical variables between patients who were treated with opioids and those who were not. An independent $t$ test was then used to compare baseline continuous variables between both groups. Multivariable logistic regression analysis was used to evaluate factors predicting opioid use; this model was adjusted for age at diagnosis, BMI, race, sex, ECOG PS, primary tumor site, and number of metastatic sites.

Kaplan-Meier survival estimates were then used to compare OS and PFS outcomes according to the use of any opioid for each disease entity. Multivariable Cox regression analysis was then used to evaluate factors predicting OS and PFS for each of the 4 disease entities. For patients with advanced pancreatic, gastric, or colorectal cancer, variables included in this model were age, BMI, race, sex, ECOG PS, opioid use, and number of metastatic sites. For patients with advanced HCC, variables included in this model included age, BMI, race, sex, ECOG PS, opioid use, extrahepatic spread, and

\section{Table 1. Specifics on the 8 Clinical Trials Included in the Current Analysis}

\begin{tabular}{|c|c|c|c|c|}
\hline Study & Start Date & End Date & $\begin{array}{l}\text { Patients Included in } \\
\text { Current Analysis } \\
\text { n (\%) }\end{array}$ & Treatment Arms \\
\hline \multicolumn{5}{|l|}{ Pancreatic cancer } \\
\hline NCT01124786 & May 2010 & November 2012 & $4,430(12.5 \%)$ & $\begin{array}{l}\text { Experimental arm: gemcitabine elaidate } \\
\text { Active comparator: gemcitabine }\end{array}$ \\
\hline NCT00844649a & March 2009 & September 2012 & 367 (10.7\%) & $\begin{array}{l}\text { Experimental arm: gemcitabine }+ \text { nab-paclitaxel } \\
\text { Active comparator: gemcitabine }\end{array}$ \\
\hline \multicolumn{5}{|l|}{ Gastric cancer } \\
\hline NCT00290966 & October 1998 & May 2003 & $218(6.3 \%)$ & $\begin{array}{l}\text { Docetaxel in combination with cisplatin or } \\
\text { docetaxel in combination with } 5-\mathrm{FU} \text { and } \\
\text { cisplatin vs combination cisplatin and } 5-\mathrm{FU}\end{array}$ \\
\hline NCT00678535 & May 2008 & March 2012 & $436(12.7 \%)$ & $\begin{array}{l}\text { Capecitabine in combination with cisplatin } \pm \\
\text { cetuximab }\end{array}$ \\
\hline \multicolumn{5}{|c|}{ Hepatocellular carcinoma } \\
\hline NCT00699374a & June 2008 & January 2013 & 544 (15.8\%) & $\begin{array}{l}\text { Sunitinib (experimental arm) vs sorafenib } \\
\text { (active comparator) }\end{array}$ \\
\hline \multicolumn{5}{|l|}{ Colorectal cancer } \\
\hline NCT00272051a & July 2002 & May 2004 & $322(9.4 \%)$ & $\begin{array}{l}\text { Experimental arm: FOLFOX and xaliproden } \\
\text { Control arm: FOLFOX and placebo }\end{array}$ \\
\hline NCT00305188a & December 2005 & October 2009 & 434 (12.5\%) & $\begin{array}{l}\text { Experimental arm: FOLFOX and xaliproden } \\
\text { Control arm: FOLFOX and placebo }\end{array}$ \\
\hline $\begin{array}{l}\text { NCT00384176 } \\
\text { Horizon III }\end{array}$ & August 2006 & August 2015 & $690(20.1 \%)$ & $\begin{array}{l}\text { Experimental arm: FOLOFOX and cediranib } \\
\text { Control arm: FOLFOX and bevacizumab }\end{array}$ \\
\hline
\end{tabular}

anly comparator arm datasets were available in the Project Data Sphere and were subsequently included in the current study cohort. 
macrovascular invasion. Variables chosen in the logistic and Cox models were based on their possible correlation with opioid intake and the possible relationship to outcomes in this patient cohort.

\section{Results}

\section{Patient Characteristics}

A total of 3,441 patients were included from the 8 clinical trials: 1,277 who received an opioid medication and 2,164 who did not. Our comparison showed that patients using opioids were more likely to be nonwhite $(P<.001)$, have an ECOG PS of $2(P<.001)$, and have the pancreas as the primary tumor site $(P<.001)$. No difference was found between both categories regarding age $(P=.124)$, BMI $(P=.145)$, or sex $(P=.490)$ (Table 2$)$. Figure 1 shows rates

\begin{tabular}{|c|c|c|c|}
\hline Characteristic & $\begin{array}{l}\text { Opioid Use } \\
(n=1,277)\end{array}$ & $\begin{array}{l}\text { No Opioids } \\
(n=2,164)\end{array}$ & $P$ Value \\
\hline Mean age (SD), y & $59.45(11.31)$ & $60.07(11.25)$ & .124 \\
\hline Mean BMI (SD), ${ }^{a} \mathrm{~kg} / \mathrm{m}^{2}$ & $24.71(4.60)$ & $24.94(4.41)$ & .145 \\
\hline Race & & & $<.001$ \\
\hline White & 904 (70.8\%) & $1,655(76.5 \%)$ & \\
\hline Nonwhite $^{b}$ & $354(27.7)$ & $501(23.2 \%)$ & \\
\hline Unknown & $19(1.5 \%)$ & $8(0.4 \%)$ & \\
\hline Sex & & & .490 \\
\hline Male & $847(66.3 \%)$ & $1,416(65.4 \%)$ & \\
\hline Female & $430(33.7 \%)$ & $746(34.5 \%)$ & \\
\hline Unknown & 0 & $2(0.1 \%)$ & \\
\hline ECOG PS & & & $<.001$ \\
\hline 0 & 409 (32.1\%) & $1,109(51.6 \%)$ & \\
\hline 1 & $834(65.5 \%)$ & $1,014(47.2 \%)$ & \\
\hline 2 & $31(2.4 \%)$ & $25(1.2 \%)$ & \\
\hline Primary tumor site & & & $<.001$ \\
\hline Pancreas & $492(38.5 \%)$ & 305 (14.1\%) & \\
\hline Gastric & $188(14.7 \%)$ & $466(21.5 \%)$ & \\
\hline Liver & 242 (19\%) & 302 (14\%) & \\
\hline Colorectal & 355 (27.8\%) & $1,091(50.4 \%)$ & \\
\hline Number of metastatic sites ${ }^{c}$ & & & $<.001$ \\
\hline 0 & $6(0.5 \%)$ & $27(1.2 \%)$ & \\
\hline 1 & $429(33.5 \%)$ & 805 (37.2\%) & \\
\hline$\geq 2$ & $600(47 \%)$ & $1,030(47.6 \%)$ & \\
\hline Unknown & 242 (19\%) & 302 (14\%) & \\
\hline
\end{tabular}

Abbreviations: BMI, body mass index; HCC, hepatocellular carcinoma; PS, performance status.

a19 patients had missing PS, and 75 patients had missing BMI

bIncluding black, Asian, and other non-Caucasian races.

cNumber of metastatic sites was not available for patients with HCC. Among patients with HCC, $47(8.6 \%)$ had evidence of macrovascular invasion and 319 (58.6\%) had extrahepatic spread.

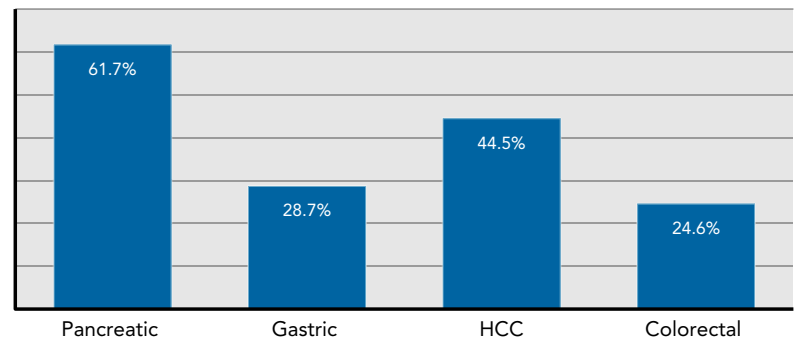

Figure 1. Rates of opioid use among patients. Abbreviation: HCC, hepatocellular carcinoma.

of opioid use among patients with different gastrointestinal cancers in the current analysis, with the highest rate seen in patients with pancreatic cancer $(61.7 \%)$ and the lowest rate in those with colorectal cancer (24.6\%). Mean follow-up for the study cohort was 11.42 months (SD, 7.34).

\section{Predictors of Opioid Use}

Multivariable logistic regression analysis suggested that the following factors predict a higher probability of

\section{Table 3. Multivariable Logistic Regression Analysis of Factors Predicting Opioid Use}

\begin{tabular}{|c|c|c|}
\hline Variable & OR $(95 \% \mathrm{Cl})$ & $P$ Value \\
\hline Age (mean; SD) & $0.990(0.984-0.997)$ & .004 \\
\hline BMI (mean; SD) & $1.006(0.989-1.024)$ & .467 \\
\hline \multicolumn{3}{|l|}{ Race $^{a}$} \\
\hline Nonwhite ${ }^{b}$ & Ref & \\
\hline White & $0.749(0.600-0.933)$ & .010 \\
\hline \multicolumn{3}{|l|}{ Sex } \\
\hline Male & Ref & \\
\hline Female & $0.936(0.796-1.102)$ & .430 \\
\hline \multicolumn{3}{|l|}{ ECOG PS } \\
\hline 0 & Ref & \\
\hline 1 & $1.751(1.490-2.058)$ & $<.001$ \\
\hline 2 & $2.135(1.185-3.848)$ & .012 \\
\hline \multicolumn{3}{|c|}{ Primary tumor site } \\
\hline Pancreas & Ref & \\
\hline Colorectal & $0.241(0.198-0.295)$ & $<.001$ \\
\hline Gastric & $0.249(0.195-0.318)$ & $<.001$ \\
\hline Liver & $0.471(0.348-0.637)$ & $<.001$ \\
\hline \multicolumn{3}{|c|}{ Number of metastatic sites ${ }^{a}$} \\
\hline 1 & Ref & \\
\hline 0 & $0.519(0.207-1.300)$ & .162 \\
\hline$\geq 2$ & $0.963(0.813-1.141)$ & .664 \\
\hline
\end{tabular}

Abbreviations: BMI, body mass index; OR, odds ratio, PS, performance status.

aPatients with unknown race and those with unknown number of metastatic sites were included in the analysis but are not reported in this table. bIncluding black, Asian, and other non-Caucasian races. 
opioid use: younger age at diagnosis (odds ratio [OR], 0.990; 95\% CI, 0.984-0.997; $P=.004$ ), nonwhite race (OR for white vs nonwhite race, 0.749 ; $95 \%$ CI, $0.600-0.933$; $P=.010$ ), higher ECOG score (OR for score 1 vs score 0 , $1.751 ; 95 \% \mathrm{CI}, 1.490-2.058 ; P<.001)$, and pancreatic primary site (OR for colorectal vs pancreatic, $0.241 ; 95 \%$ CI, 0.198-0.295; $P<.001$ ) (Table 3).

\section{Survival Outcomes in the Current Study}

Using Kaplan-Meier survival estimates, we found that receiving opioids was consistently associated with worse OS $(P=.008$ for pancreatic cancer; $P<.001$ for gastric cancer, HCC, and colorectal cancer) (Figure 2A-D).

\section{A}

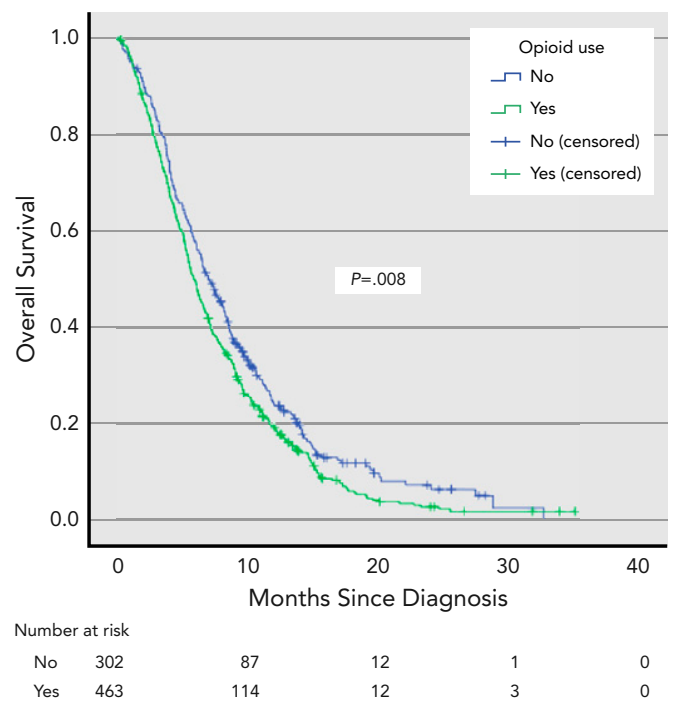

C

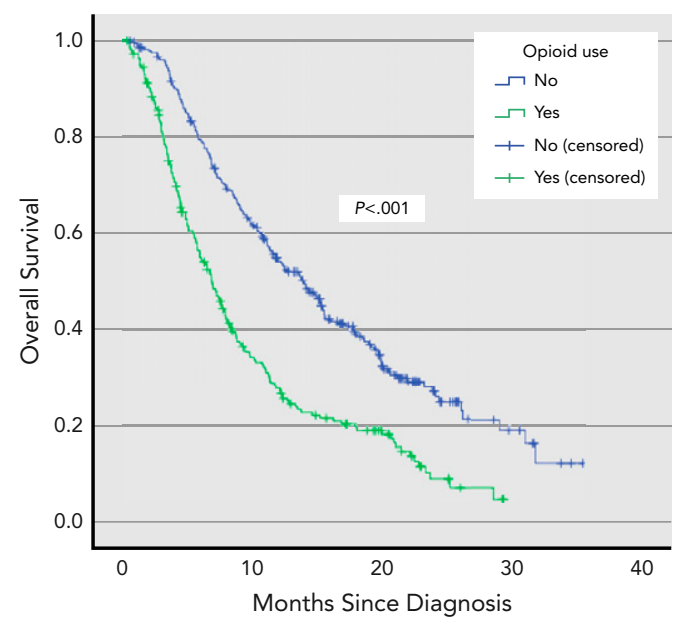

Number at risk

$\begin{array}{rrrrrr}\text { No } & 275 & 160 & 53 & 8 & 0 \\ \text { Yes } & 218 & 63 & 23 & 0 & 0\end{array}$

Opioid use was associated with worse PFS among patients with pancreatic $(P=.009)$, gastric $(P=.002)$, and colorectal cancers $(P=.018)$, but not among those with HCC $(P=.106)$ (Figure 3A-D).

Adjusted multivariable Cox regression analysis among patients with different disease entities showed that opioid use was associated with worse OS among patients with pancreatic cancer (hazard ratio [HR], 1.245; 95\% CI, 1.063-1.459; $P=.007$ ), gastric cancer (HR, 1.725; 95\% CI, 1.403-2.122; $P<.001$ ), HCC (HR, 1.841; 95\% CI, $1.480-2.290 ; P<.001$ ), and colorectal cancer (HR, 1.651; 95\% CI, 1.380-1.975; $P<.001)$. Adjusted multivariable analysis of PFS among patients with different disease

B

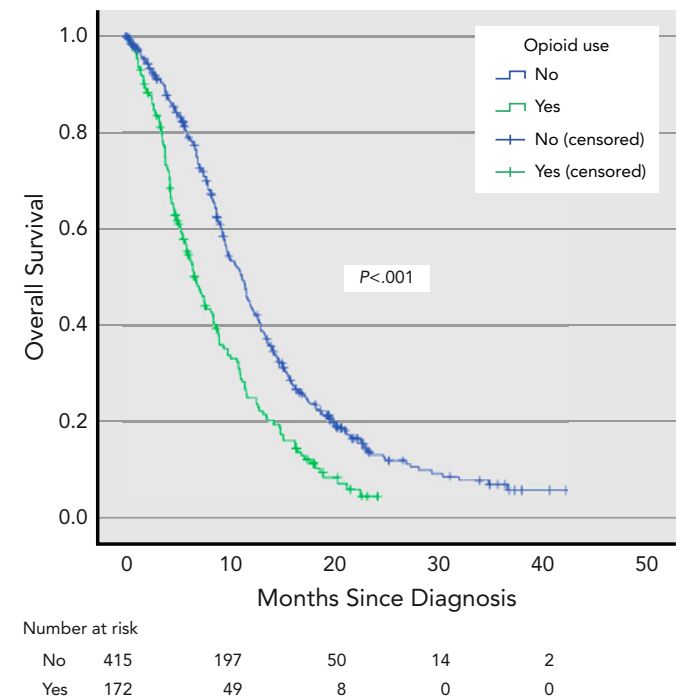

D

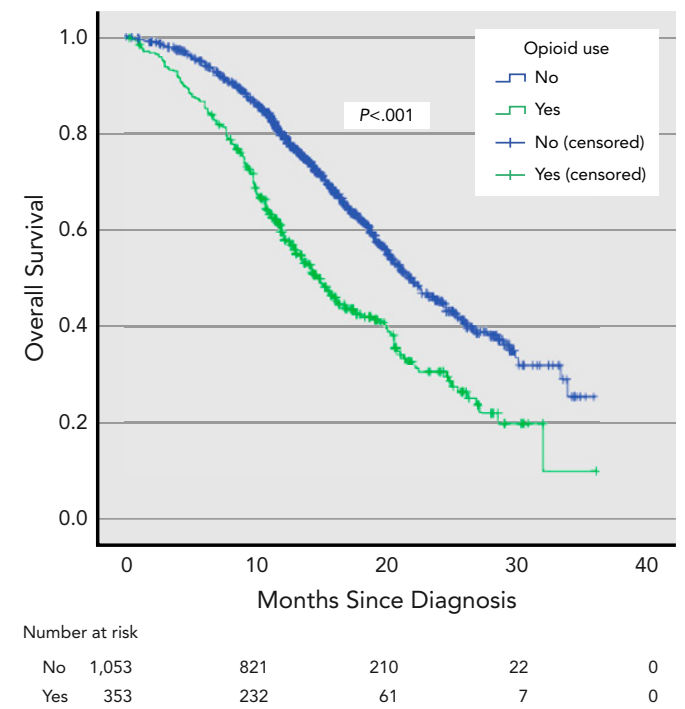

Figure 2. Overall survival according to opioid use among patients with (A) pancreatic cancer, (B) gastric cancer, (C) hepatocellular carcinoma, and (D) colorectal cancer. 
A

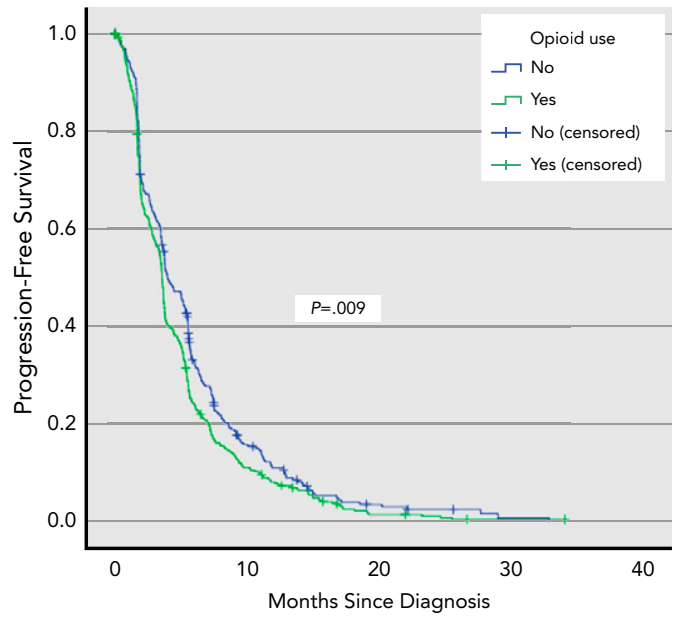

Number at risk

$\begin{array}{ll}\text { No } & 302 \\ \text { Yes } & 473\end{array}$

C

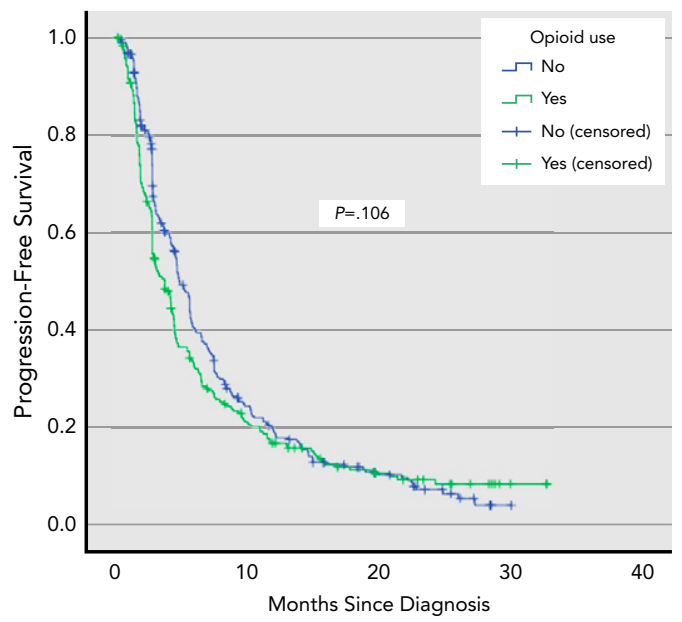

Number at risk

$\begin{array}{ll}\text { No } & 300 \\ \text { Yes } & 242\end{array}$

Figure 3. Progression-free survival according to opioid use among patients with (A) pancreatic carcinoma, (B) gastric cancer, (C) hepatocellular carcinoma, and (D) colorectal cancer.

entities showed that opioid use was associated with worse outcomes among patients with pancreatic cancer (HR, 1.216; 95\% CI, 1.043-1.416; $P=.012$ ) and gastric cancer (HR, 1.277; 95\% CI, 1.056-1.544; $P=.012$ ) but not among patients with HCC (HR, 1.071; 95\% CI, $0.887-1.294 ; P=.475)$ or colorectal cancer (HR, 1.132; 95\% CI, 0.983-1.303; $P=.085$ ) (Table 4).

\section{Discussion}

The aim of this study was to evaluate the patterns and impact of opioid use among patients with advanced
B

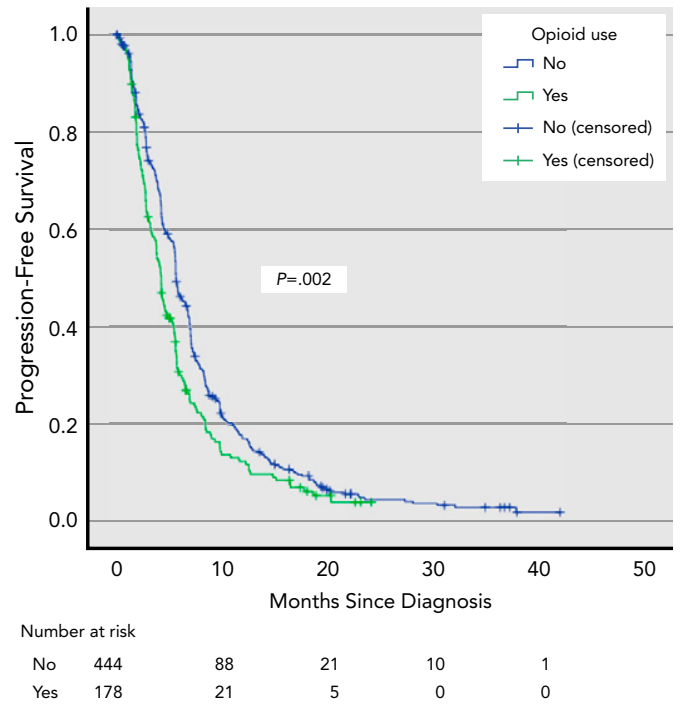

D

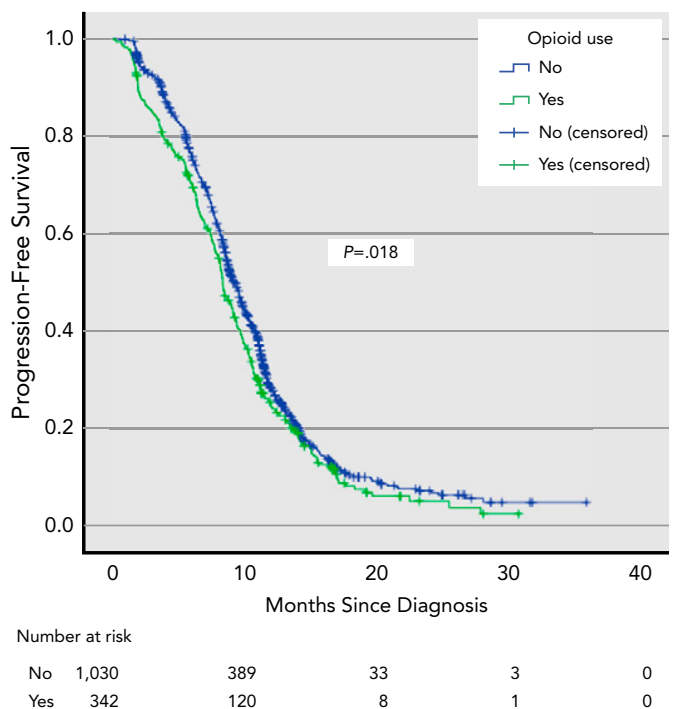




\begin{tabular}{|c|c|c|c|c|}
\hline \multirow[b]{2}{*}{ Disease } & \multicolumn{2}{|c|}{ Overall Survival } & \multicolumn{2}{|c|}{ Progression-Free Survival } \\
\hline & HR $(95 \%$ Cl) & $P$ Value & HR (95\% Cl) & $P$ Value \\
\hline \multicolumn{5}{|l|}{ Pancreatic cancer ${ }^{\mathrm{a}}$} \\
\hline No opioid use & Ref & & Ref & \\
\hline Opioid use & $1.245(1.063-1.459)$ & .007 & $1.216(1.043-1.416)$ & .012 \\
\hline \multicolumn{5}{|l|}{ Gastric cancer $^{a}$} \\
\hline No opioid use & Ref & & Ref & \\
\hline Opioid use & $1.725(1.403-2.122)$ & $<.001$ & $1.277(1.056-1.544)$ & .012 \\
\hline \multicolumn{5}{|c|}{ Hepatocellular carcinomab } \\
\hline No opioid use & Ref & & Ref & \\
\hline Opioid use & $1.841(1.480-2.290)$ & $<.001$ & $1.071(0.887-1.294)$ & .475 \\
\hline \multicolumn{5}{|l|}{ Colorectal cancer ${ }^{\mathrm{a}}$} \\
\hline No opioid use & Ref & & Ref & \\
\hline Opioid use & $1.651(1.380-1.975)$ & $<.001$ & $1.132(0.983-1.303)$ & .085 \\
\hline
\end{tabular}

Abbreviation: HR, hazard ratio.

adjusted for age, race, sex, ECOG performance status, body mass index, and number of metastatic sites.

${ }^{b}$ Adjusted for age, race, sex, ECOG performance status, body mass index, vascular invasion, and extrahepatic spread.

shown to have protumor effects in preclinical studies. ${ }^{14}$ These protumor effects have been partly linked to their inhibition of humoral and cell-mediated immunity and their proangiogenic effects. ${ }^{15,16}$ Along the lines of the current study, an interesting post hoc analysis of 2 randomized controlled studies evaluated the impact of methylnaltrexone (a peripherally acting $\mu$-opioid receptor antagonist used to treat opioid-induced constipation) on the survival of patients with advanced cancer. This study has suggested that treatment using this agent (and, more so, response to this drug) is associated with improved survival. ${ }^{17}$ A second explanation might relate to the correlation between pain and poor survival outcomes among patients with different solid tumors. ${ }^{18,19}$ The presence of pain may be associated with more advanced local or metastatic disease, and thus poorer OS. A third explanation might be related to the fact that severe pain itself has been correlated with poor OS in patients with advanced cancer ${ }^{20}$ Opioid use might be only a surrogate marker for that severe pain. Notably, patients who used opioids in our study were more likely to have a high ECOG PS. This supports the assumption that opioid use is a culprit of poor patient-/diseaserelated factors rather than the real underlying reason for poorer survival.

It is also notable that opioid use has been linked to poor survival among patient populations without cancer. $^{21,22}$ Thus, it is possible that a negative relationship between opioid use and survival does exist outside of cancer-mediated pathways.

Our study has several limitations. First, opioid use was not the primary research question in any of the included trials. Thus, although the current study is based on a prospectively collected dataset, the research question is retrospective. Second, this pooled analysis spans 4 different cancers, and therefore there is heterogeneity in the current dataset. To mitigate the impact of this heterogeneity, survival analyses were conducted for each disease entity independently. Third, the included clinical trial datasets reported information about opioid use during first-line systemic treatment without providing detailed information about subsequent opioid use. Fourth, although the included clinical trials reported data regarding opioid use among patients with advanced gastrointestinal cancer, they did not clarify the degree or form of pain these patients experienced. Thus, it is not possible to isolate the prognostic impact of opioids from the prognostic impact of pain on survival outcomes in the studied cohort. These limitations need to be weighed against the clear strengths of the current analysis. Most important is the reliance on clinical trial datasets in generating this pooled analysis. This should indicate greater credibility of the results of the current analysis than those in previously conducted retrospective studies evaluating the same research question.

Furthermore, it is important to note that the current study evaluated patients recruited within the context of a clinical trial. Thus, included patients are more likely to have less comorbidity and better PS than the general population. Caution should therefore be exercised when generalizing the results of the current analysis to real-world settings.

Findings of the current study have several implications for clinical practice and research directions. 
Although opioid analgesia can be an indispensable part of the analgesic regimen for some patients with advanced gastrointestinal cancer and intractable pain, physicians should not rush to use it in patients with milder pain symptoms that can be controlled with other pharmacologic or nonpharmacologic interventions (eg, local radiotherapy for bone metastases). Although our study suggests an association between opioid use and poorer outcomes, it falls short of establishing causation. Future research should focus on the potential mechanisms underlying opioid-induced cancer progression and whether this is universal to all opioid analgesics or pertinent only to certain subtypes; the impact of specific types/doses of opioids on oncologic outcomes in the current dataset cannot be assessed, due to the small number of patients receiving individual opioid medications.

\section{Conclusions}

Results of our study suggest that opioid use is consistently associated with worse OS among patients with different gastrointestinal cancers. Whether this results from a genuine negative impact of opioid use on survival or opioid use serves merely as the surrogate marker for poor patient-/disease-related conditions is unclear. Further studies are needed to study the mechanisms underlying this observation.

Submitted September 26, 2019; accepted for publication November 26, 2019.

Author contributions: Study concept: All authors. Data curation and formal analysis: Abdel-Rahman. Visualization and writing-original draft: AbdelRahman. Writing-review and editing: All authors.

Disclosures: The authors have disclosed that they have not received any financial considerations from any person or organization to support the preparation, analysis, results, or discussion of this article.

Disclaimer: This publication is based on research using information obtained from www.projectdatasphere.org, which is maintained by Project Data Sphere, LLC. Neither Project Data Sphere, LLC, nor the owner(s) of any information from the website have contributed to, approved, or are in any way responsible for the contents of this publication. All procedures were performed according to the ethical standards of the institutional and/or national research committee and the 1964 Helsinki declaration and its later amendments or comparable ethical standards. Informed consent was obtained from all participants included in the study.

Correspondence: Omar Abdel-Rahman, MD, Department of Oncology, University of Alberta, 11560 University Avenue, Edmonton, Alberta, Canada T4G 1Z2. Email: omar.abdelsalam@ahs.ca

\section{References}

1. Hinther A, Abdel-Rahman O, Cheung WY, et al. Chronic postoperative opioid use: a systematic review. World J Surg 2019;43:2164-2174.

2. Ballantyne JC. Chronic pain following treatment for cancer: the role of opioids. Oncologist 2003;8:567-575.

3. Zajączkowska R, Leppert W, Mika J, et al. Perioperative immunosuppression and risk of cancer progression: the impact of opioids on pain management. Pain Res Manag 2018;2018:9293704.

4. Aich A, Gupta P, Gupta K. Could perioperative opioid use increase the risk of cancer progression and metastases? Int Anesthesiol Clin 2016;54: e1-16.

5. Oh TK, Jeon JH, Lee JM, et al. Investigation of opioid use and long-term oncologic outcomes for non-small cell lung cancer patients treated with surgery. PLoS One 2017;12:e0181672.

6. Maher DP, Wong W, White PF, et al. Association of increased postoperative opioid administration with non-small-cell lung cancer recurrence: a retrospective analysis. Br J Anaesth 2014;113(Suppl 1):i88-94.

7. CEO Roundtable on Cancer Life Sciences Consortium. Project Data Sphere, LLC. Accessed September 19, 2019. Available at: https://www. ceoroundtableoncancer.org/programs/project-data-sphere-llc

8. Poplin E, Wasan H, Rolfe L, et al. Randomized, multicenter, phase II study of CO-101 versus gemcitabine in patients with metastatic pancreatic ductal adenocarcinoma: including a prospective evaluation of the role of hENT1 in gemcitabine or CO-101 sensitivity. J Clin Oncol 2013;31: 4453-4461.

9. Von Hoff DD, Ervin T, Arena FP, et al. Increased survival in pancreatic cancer with nab-paclitaxel plus gemcitabine. N Engl J Med 2013;369: 1691-1703.

10. Van Cutsem E, Moiseyenko VM, Tjulandin S, et al Phase III study of docetaxel and cisplatin plus fluorouracil compared with cisplatin and fluorouracil as first-line therapy for advanced gastric cancer: a report of the V325 Study Group. J Clin Oncol 2006;24:4991-4997

11. Lordick F, Kang YK, Chung HC, et al. Capecitabine and cisplatin with or without cetuximab for patients with previously untreated advanced gastric cancer (EXPAND): a randomised, open-label phase 3 trial. Lancet Oncol 2013;14:490-499.
12. Cheng $A L$, Kang $Y K$, Lin DY, et al. Sunitinib versus sorafenib in advanced hepatocellular cancer: results of a randomized phase III trial. J Clin Oncol 2013;31:4067-4075.

13. Schmoll HJ, Cunningham D, Sobrero A, et al. Cediranib with mFOLFOX6 versus bevacizumab with mFOLFOX6 as first-line treatment for patients with advanced colorectal cancer: a double-blind, randomized phase III study (HORIZON III). J Clin Oncol 2012;30:3588-3595.

14. Lee BM, Cata JP. Impact of anesthesia on cancer recurrence. Rev Esp Anestesiol Reanim 2015;62:570-575.

15. Cakmakkaya OS, Kolodzie K, Apfel CC, et al. Anaesthetic techniques for risk of malignant tumour recurrence. Cochrane Database Syst Rev 2014 CD008877.

16. Boland JW, Pockley AG. Influence of opioids on immune function in patients with cancer pain: from bench to bedside. Br J Pharmacol 2018, 175:2726-2736

17. Janku F, Johnson LK, Karp DD, et al. Treatment with methylnaltrexone is associated with increased survival in patients with advanced cancer. Ann Oncol 2016;27:2032-2038.

18. Abdel-Rahman O. Prognostic impact of baseline quality of life status among patients with advanced gastric cancer; results from two randomized studies. Expert Rev Pharmacoecon Outcomes Res 2019;19:711-715.

19. Zylla D, Kuskowski MA, Gupta K, et al. Association of opioid requirement and cancer pain with survival in advanced non-small cell lung cancer. $\mathrm{Br} \mathrm{J}$ Anaesth 2014;113(Suppl 1):i109-116.

20. Woopen H, Richter R, Inci G, et al. The prognostic and predictive role of pain before systemic chemotherapy in recurrent ovarian cancer: an individual participant data meta-analysis of the North-Eastern German Society of Gynecological Oncology (NOGGO) of 1226 patients. Support Care Cancer 2020;28:1997-2003.

21. Ray WA, Chung CP, Murray KT, et al. Prescription of long-acting opioids and mortality in patients with chronic noncancer pain. JAMA 2016;315: 2415-2423.

22. Oh TK, Jeon YT, Choi JW. Trends in chronic opioid use and association with five-year survival in South Korea: a population-based cohort study. $\mathrm{Br}$ J Anaesth 2019;123:655-663. 